\title{
POSH is an Intracellular Signal Transducer for the Axon Outgrowth Inhibitor Nogo66
}

\author{
Heather M. Dickson, ${ }^{1}$ Jonathan Zurawski, ${ }^{1}$ Huanqing Zhang, ${ }^{2}$ David L. Turner, ${ }^{1,2}$ and Anne B. Vojtek ${ }^{1}$ \\ ${ }^{1}$ Department of Biological Chemistry and ${ }^{2}$ Molecular and Behavioral Neuroscience Institute, University of Michigan, Ann Arbor, Michigan 48109
}

\begin{abstract}
Myelin-derived inhibitors limit axon outgrowth and plasticity during development and in the adult mammalian CNS. Nogo66, a functional domain of the myelin-derived inhibitor NogoA, signals through the PirB receptor to inhibit axon outgrowth. The signaling pathway mobilized by Nogo66 engagement of PirB is not well understood. We identify a critical role for the scaffold protein Plenty of SH3s (POSH) in relaying process outgrowth inhibition downstream of Nogo66 and PirB. Blocking the function of POSH, or two POSH-associated proteins, leucine zipper kinase (LZK) and Shroom3, with RNAi in cortical neurons leads to release from myelin and Nogo66 inhibition. We also observed autocrine inhibition of process outgrowth by NogoA, and suppression analysis with the POSH-associated kinase LZK demonstrated that LZK operates downstream of NogoA and PirB in a POSH-dependent manner. In addition, cerebellar granule neurons with an RNAi-mediated knockdown in POSH function were refractory to the inhibitory action of Nogo66, indicating that a POSHdependent mechanism operates to inhibit axon outgrowth in different types of CNS neurons. These studies delineate an intracellular signaling pathway for process outgrowth inhibition by Nogo66, comprised of NogoA, PirB, POSH, LZK, and Shroom3, and implicate the POSH complex as a potential therapeutic target to enhance axon outgrowth and plasticity in the injured CNS.
\end{abstract}

\section{Introduction}

The regenerative and plastic capacity of the adult mammalian CNS is limited, contributing to poor functional recovery after injury or disease. CNS axons are capable of regenerating but fail to do so, in part because factors present in the CNS actively prevent axon outgrowth (Kubo et al., 2007; Walmsley and Mir, 2007; Gonzenbach and Schwab, 2008; Schwab and Caroni, 2008). Factors that limit axon outgrowth and plasticity have been purified from CNS myelin and include myelin-associated glycoprotein (MAG), oligodendrocyte myelin glycoprotein, and NogoA (McGee et al., 2005; Walmsley and Mir, 2007; Giger et al., 2008; Gonzenbach and Schwab, 2008). In addition to limiting axon outgrowth and plasticity in the adult CNS, NogoA also regulates axon outgrowth and plasticity during development (McGee et al., 2005; Syken et al., 2006; Mingorance-Le Meur et al., 2007; Atwal et al., 2008; Wang et al., 2008; Brösamle and Halpern, 2009; Datwani et al., 2009; Petrinovic et al., 2010).

Nogo66, an axon outgrowth inhibitory domain of NogoA, engages cell surface receptors Nogo66 Receptor 1 (NgR1) and Paired Ig-like Receptor B (PirB) to mediate intracellular signal transduction (GrandPré et al., 2000; Fournier et al., 2001; Oertle et al., 2003; Atwal et al., 2008). NgR1 links to Rho and its downstream effector Rho kinase to regulate cytoskeletal dynamics as-

Received March 15, 2010; revised July 22, 2010; accepted Aug. 7, 2010.

This work was supported by National Institute of Mental Health Grant MH-073085 (to A.B.V.), National Institute of Aging Grant AG-034264 (to A.B.V.), National Institute of Health Grant NS-38698 (to D.L.T.), and the Biomedical Research Council, University of Michigan (to A.B.V.).

The University of Michigan has a patent on miR-155-based RNAi technology. A.B.V. and D.L.T. are potential recipients of royalties paid to the University of Michigan for licensed use.

Correspondence should be addressed to Dr. Anne B. Vojtek, Department of Biological Chemistry, University of Michigan, 3301C MSRB III; 1150 W. Medical Center Drive, Ann Arbor, MI 48109-5606. E-mail: avojtek@umich.edu. DOI:10.1523/JNEUROSCI.1324-10.2010

Copyright $\odot 2010$ the authors $\quad 0270-6474 / 10 / 3013319-07 \$ 15.00 / 0$ sociated with growth cone collapse and inhibition of axon outgrowth (Walmsley and Mir, 2007; Gonzenbach and Schwab, 2008). PirB is a recently identified receptor for myelin-derived inhibitory substrates (Syken et al., 2006; Atwal et al., 2008). The signaling pathway activated when myelin-derived inhibitors engage PirB is not known. However, in hematopoietic cells, where PirB signaling has been more extensively studied, phosphorylation of the receptor at specific tyrosines by src family kinases recruits SH2-homology-containing protein tyrosine phosphatases SHP1 and SHP2 (Kubagawa et al., 1997; Ho et al., 1999).

Plenty of SH3s (POSH, also known as SH3rf1) is an intracellular, multidomain scaffold protein that regulates diverse biological functions, including apoptosis, calcium homeostasis, morphogenesis, and neuronal process outgrowth (Tapon et al., 1998; Schnorr et al., 2001; Figueroa et al., 2003; Xu et al., 2003, 2005; Tuvia et al., 2007; Taylor et al., 2008). POSH contains multiple protein-protein interaction domains, including an N-terminal RING domain, four Src homology 3 (SH3) domains, and a Rac binding domain. As a scaffold protein, $\mathrm{POSH}$ exerts its function through interacting partners, which include the actinmyosin regulatory protein Shroom3, the small GTPase Rac, and mixed lineage kinases (Tapon et al., 1998; Xu et al., 2003; Taylor et al., 2008; Zhang et al., 2009). We previously reported that POSH limits axon growth through a Shroom3-ROCK-myosin signaling pathway (Taylor et al., 2008). Here, we demonstrate that the scaffold protein POSH, in association with Shroom 3 and the mixed lineage kinase LZK, relays axon outgrowth inhibition downstream of NogoA and PirB.

\section{Materials and Methods}

Antibodies. For axon outgrowth assays, cells were stained with anti-green fluorescent protein (GFP) rabbit primary antibody and Alexa Fluor 488 goat anti-rabbit secondary antibody (both from Invitrogen). For West- 
ern blots, the following primary antibodies were used: goat anti-Nogo (N18) and anti-ILT-5/PirB (C-19) (Santa Cruz Biotechnology), rabbit anti-actin (Sigma), and rabbit anti-GFP (Invitrogen). Secondary antibodies used were goat anti-rabbit IgG horseradish peroxidase (HRP) conjugate (Bio-Rad) and bovine anti-goat IgG HRP (Santa Cruz Biotechnology).

Expression constructs and RNAi. pUI4-SIBR-GFP is a short interfering RNA (siRNA) expression vector that coexpresses the GFP protein and an siRNA from an intronic expression cassette (the SIBR cassette) based on the miR-155 microRNA precursor (Chung et al., 2006; Taylor et al., 2008). For each siRNA, a pUI4-SIBR-GFP vector expressing one to four identical tandem copies of the siRNA SIBR cassette was constructed (Chung et al., 2006; Taylor et al., 2008). POSH-6, luciferase (a functional control RNAi vector), Shroom3-3, and myosinIIA-1 are described by Taylor et al. (2008). The sequences of LZK, Nogo, and PirB siRNAs are as follows: LZK-1, 5' UUCAUCGGGACUGUUCGAGUGG 3'; LZK-2, 5' AUCAAUGUUACAGUAGCCGGAG $3^{\prime}$; Nogo-1, $5^{\prime}$ AAUCUUUGAAAUGACGGUUACG 3'; Nogo-3, 5' UAUACCGUCAUAACUAACUGGA 3'; PirB-294, 5' AACAAUAACAGCGAUAUUGCCC $3^{\prime}$; and PirB-874 siRNA, 5' AACAUCGAUAUUGACCUGCAUU $3^{\prime}$. Western blot analysis confirmed knock-down of endogenous NogoA and PirB (supplemental Fig. S4, available at www.jneurosci.org as supplemental material). To construct CS2+NFLAG LZK, an N-terminally FLAG-tagged construct, LZK was cloned by reverse transcription (RT)-PCR from RNA isolated from adult mouse brain. Kinase dead LZK was constructed by site-directed mutagenesis, AAG being converted to GCG, substituting lysine 195 to alanine. ATP fails to bind at the active site in the mutant protein, resulting in a catalytically defective, kinase-dead mutant (Ikeda et al., 2001).

Preparation of myelin and recombinant proteins. Myelin extracts were prepared from adult rat brains, as described previously (Larocca and Norton, 2006). His-SUMO-conjugated Nogo66 (amino acids 1055-1079) or His-SUMO was expressed overnight at $25^{\circ} \mathrm{C}$ in Escherichia coli and purified on Ni-NTA His bind resin (Qiagen). Briefly, E. coli were lysed by sonication in PBS+ (PBS, $0.1 \mathrm{~mm}$ PMSF, $0.35 \mathrm{mg}$ of aprotinin, $0.1 \% \beta$-mercaptoethanol, $10 \mathrm{~mm}$ imidazole, 2 nм leupeptin). Triton $\mathrm{X}-100$ was added to the lysate at $1 \%$ of the final volume. Lysates were incubated with Ni-NTA His bind resin for $1 \mathrm{~h}$ at $4^{\circ} \mathrm{C}$ and washed three times in PBS + with $300 \mathrm{~mm} \mathrm{NaCl}$. Protein was eluted from the beads with elution buffer ( $50 \mathrm{~mm} \mathrm{NaHPO}_{4}, 300 \mathrm{~mm} \mathrm{NaCl}, 250 \mathrm{~mm}$ imidazole) and $25 \%$ glycerol was added. Protein concentration was determined by Bradford assay (Bio-Rad) and Coomassie gel with bovine serum albumin standards.

Axon outgrowth assays. Four-well chamber slides (Lab Tek II, Fisher Scientific) were coated with $10 \mu \mathrm{g} / \mathrm{ml}$ poly-L-lysine for $4 \mathrm{~h}$ then overnight with $2 \mu \mathrm{g} / \mathrm{ml}$ laminin (Invitrogen), or laminin plus myelin at $4^{\circ} \mathrm{C}$, or laminin plus control His-SUMO $\left(2.5 \mu \mathrm{g} / \mathrm{cm}^{2}\right)$, or laminin plus HisSUMO Nogo66 $\left(2.5 \mu \mathrm{g} / \mathrm{cm}^{2}\right)$. After overnight incubation, unbound substrates were removed by rinsing with PBS. Cortical primary progenitors were cultured as previously described (Taylor et al., 2008). Primary progenitors were nucleofected with a total of $6 \mu \mathrm{g}$ of DNA $(4.5 \mu \mathrm{g}$ of pUI4 vector and $1.5 \mu \mathrm{g}$ of empty vector control, pCS2-NFLAG LZK, or pCS2-NFLAG LZK KD, or $3 \mu \mathrm{g}$ of pUI4 vector and $3 \mu \mathrm{g}$ of pUI4-myosin IIA RNAi expression vector). Cells were fixed in $3.7 \%$ formaldehyde $72 \mathrm{~h}$ postnucleofection. Cells were stained with an anti-GFP primary antibody and Alexa Fluor 488 goat anti-rabbit secondary antibody (both from Invitrogen). The efficiency of conucleofection of two different plasmids in primary cortical neurons is $94 \%$. Conucleofection efficiency was determined by nucleofecting two plasmids expressing different markers (mCherry or GFP) and the percentage of cells expressing GFP, mCherry, or both markers was determined in two independent experiments.

Measurement of process length. The length of the longest process per cell was measured in photographs of fixed, GFP-stained neurons with the polyline function in MicroSuite imaging software version 5.0 (Olympus) (Taylor et al., 2008). Processes $50 \mu \mathrm{m}$ (three times the length of the cell body) or greater were measured. In the myelin and Nogo66 inhibition assays, all processes were measured, including processes less than $50 \mu \mathrm{m}$. Statistical significance was assessed using the Student's $t$ test. The datasets analyzed in the $t$ test were the measurements of the total number of GFP-positive neurons from three independent nucleofections.

Cerebellar granule neurons. Cerebellar granule neurons (CGNs) were isolated from the cerebellum of postnatal d 8 mice as described previously (Bilimoria and Bonni, 2008). CGNs were nucleofected as described previously (Venkatesh et al., 2005; Robak et al., 2009) with a total of $4 \mu \mathrm{g}$ of DNA [luciferase (control RNAi vector) or POSH-6 RNAi vector]. Cells were plated onto four-well chamber slides and incubated for $4 \mathrm{~h}$ with 10 $\mu \mathrm{g} / \mathrm{ml}$ poly-L-lysine, then overnight with $2 \mu \mathrm{g} / \mathrm{ml}$ laminin or laminin plus myelin at room temperature, laminin plus control His-SUMO (2.5 $\left.\mu \mathrm{g} / \mathrm{cm}^{2}\right)$, or laminin + His-SUMO Nogo66 $\left(2.5 \mu \mathrm{g} / \mathrm{cm}^{2}\right)$ at $4^{\circ} \mathrm{C}$. Twentyfour hours later, postnucleofection cells were fixed with $3.7 \%$ formaldehyde. Cells were incubated with rabbit anti-GFP primary antibody (Invitrogen) and neuronal class III $\beta$-tubulin monoclonal antibody TuJ1 (Covance), followed by detection with Alexa Fluor 488 goat anti-rabbit and Alexa Fluor 594 goat anti-mouse secondary antibodies (both from Invitrogen). Average process length was quantified as described above and by Taylor et al. (2008).

$R T-P C R$. RNA was purified from untransfected cortical neuron cultures (RNeasy kits; Qiagen) and genomic DNA was removed from RNA samples using RNase-Free DNase (Qiagen). RT was performed using SuperScript II (Invitrogen) and Random Primer 12 (NEB) at $25^{\circ} \mathrm{C}$ for 10 min, $42^{\circ} \mathrm{C}$ for $1 \mathrm{~h}$, and $72^{\circ} \mathrm{C}$ for $15 \mathrm{~min}$. cDNA samples were analyzed by PCR (Expand High Fidelity PCR System; Roche) with the following primers: POSH F1, 5' CAGGTCCATATAAGCACCACTG 3'; POSH R1, 5' GGTAGGGGACATCTGAAGGGA 3'; POSH F2, 5' GTGACTAAAGAGCACAAAGCAG 3'; POSH R2, 5' CAAGGCACACTTTACACATCAG 3'; Nogo F1, 5' GTGCCCTTATTGCTTCCAAA 3'; Nogo R3, 5' TCTGGATAGCTTGGATCACACCCTTA 3'; Nogo F3 5' CAGGGGCTCGGGCTCAGT $3^{\prime}$; Nogo F-C $5^{\prime}$ ATGGACGATCAGAAGAAACGTTGGAA 3'; HPRT forward, 5' CAAACTTTGCTTTCCCTGGT 3'; HPRT reverse, 5' CAAGGGCATATCCAACAACA 3'. Nogo F1, Nogo F3, and Nogo R3 have been previously described (Makeyev et al., 2007). PCR products were analyzed by agarose gel electrophoresis. Similar results were obtained with two independent RNA samples.

Western blot analysis to test efficacy of RNAi constructs. Western blot analysis was performed to test the ability of the siRNAs to target endogenous Nogo. Cellular extracts were prepared from transiently transfected, puromycin-selected P19 cells, as previously described (Vojtek et al., 2003; Taylor et al., 2008). To test the efficacy of the vector expressed PirB siRNAs, HEK 293 cells were transfected with a PirB expression construct together with a control or PirB RNAi vector. Western analysis was performed on the extracts prepared $36 \mathrm{~h}$ after transfection.

\section{Results}

In a previous report, we demonstrated that POSH limits axon outgrowth through a Shroom3-ROCK-myosin signaling pathway (Taylor et al., 2008). To determine whether myelin inhibitors signal to POSH to limit axon outgrowth, control neurons or neurons with $\mathrm{RNAi}$-mediated knockdown of POSH function were assessed for their ability to extend processes in the presence of CNS myelin. Mouse primary embryonic cortical neurons were nucleofected with control or POSH RNAi expression vectors and cultured on poly-L-lysine/laminin (PLL) with or without purified myelin. The UI4-SIBR RNAi vectors express a single vectorderived transcript for RNAi and GFP expression, with GFP expression identifying the transfected neurons (Chung et al., 2006; Taylor et al., 2008). Process outgrowth in GFP-labeled cortical neurons was analyzed $72 \mathrm{~h}$ after nucleofection and plating. Myelin reduced average process length of control RNAi-transfected neurons to $66 \%$ of the length on PLL only (Fig. $1 A, B, G$ ). POSH RNAi neurons exhibited increased process length relative to control RNAi neurons when plated on PLL, as we previously reported (Taylor et al., 2008), indicating that POSH negatively regulates process outgrowth (Fig. $1 A, D, G$ ). Strikingly, process length of POSH RNAi neurons was not reduced when plated on myelin (Fig. $1 D, E, G$ ). The observation that POSH RNAi neurons 

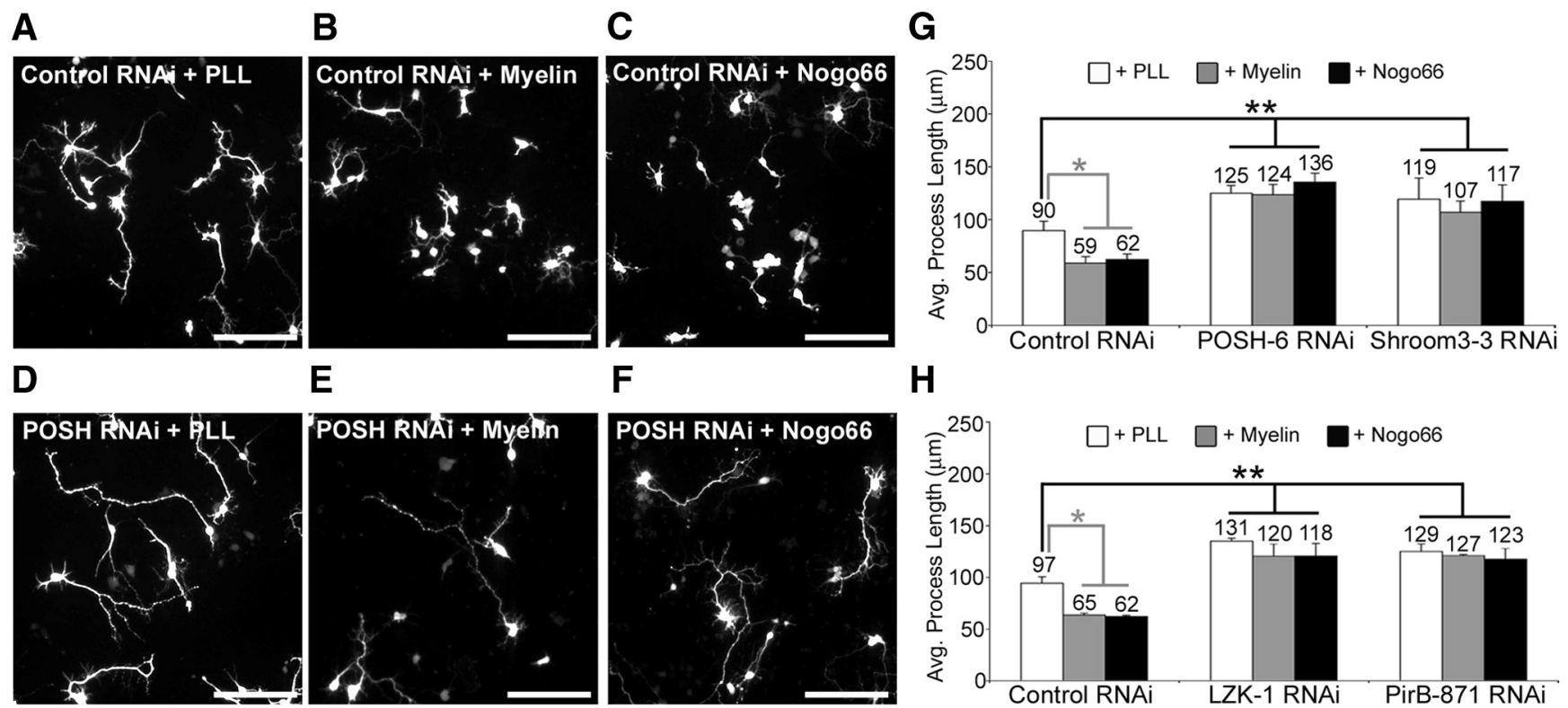

\section{E}

\section{$\mathbf{F}$}
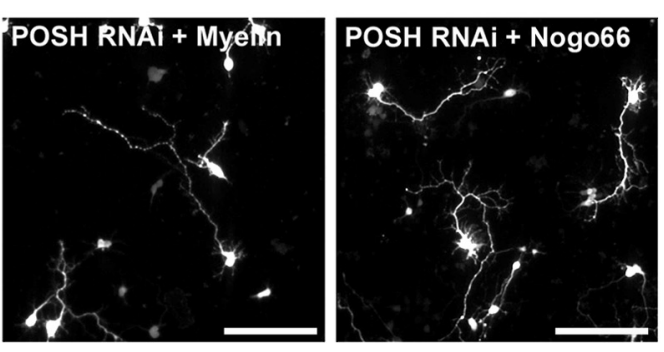

\section{$\mathbf{H}$}

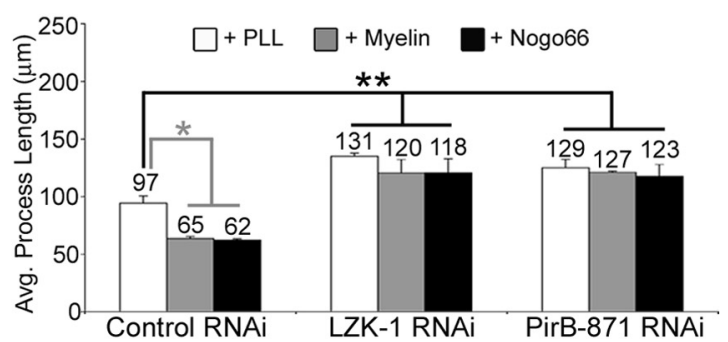

Figure 1. Primary cortical neurons with RNAi knockdown of POSH, POSH-associated proteins, or the PirB receptor are refractory to myelin and Nogo66-mediated inhibition of axon outgrowth. $\boldsymbol{A}-\boldsymbol{H}$, Cortical neurons (embryonic day 14.5) were nucleofected with the indicated RNAi expression vectors and plated to PLL- $(\boldsymbol{A}, \boldsymbol{D})$, PLL plus myelin- (B, E), or PLL plus Nogo66- (C, $\boldsymbol{F})$ coated dishes. Neurons were fixed $3 \mathrm{~d}$ after nucleofection, stained for GFP, and process length was determined. RNAi vectors express GFP and a specific siRNA from a single vector-derived transcript. The control RNAi vector expresses a functional siRNA that targets luciferase and serves as a control for nonspecific effects. POSH-6, Shroom3-3, LZK-1, and PirB-871 RNAi vectors express specific siRNAs that target POSH, Shroom3, LZK, and PirB receptor mRNA sequences, respectively. Scale bars, $100 \mu \mathrm{m} . \mathbf{G}, \boldsymbol{H}$, Average process length was determined from three independent experiments, with 551-810 neurons in total measured per condition. Myelin and Nogo66 reduced average process length of control neurons. In contrast, neither myelin nor Nogo66 reduced average process length of P0SH, Shroom3, LZK, or PirB-RNAi knockdown neurons. ${ }^{*},{ }^{* *} p<0.0001$; Student's $t$ test.

are refractory to inhibition by myelin suggests that, in primary cortical neurons, myelin-based inhibitors act through a signaling pathway that includes POSH.

Several proteins in myelin limit process outgrowth, including NogoA (Giger et al., 2008; Gonzenbach and Schwab, 2008). We tested whether Nogo66, a soluble domain of the NogoA ligand, inhibits process outgrowth of control or POSH RNAi neurons. Primary cortical neurons were nucleofected with control or POSH RNAi expression vectors and cultured on bacterially expressed and purified Nogo66. Process length was assessed in fixed, GFP-stained neurons. Nogo66 inhibited process length of control RNAi neurons (Fig. 1 $A, C, G$ ). In contrast, POSH RNAi neurons were refractory to the inhibitory action of Nogo66 (Fig. $1 D, F, G)$. This result suggests that Nogo66 signals to POSH to inhibit process outgrowth and that the inhibitory action of myelin is mediated, at least in part, through a NogoA/POSH signaling pathway. Nogo66 also inhibits axon outgrowth in CGNs (K. C. Wang et al., 2002; Atwal et al., 2008). Since different neuronal cell types could use different mechanisms for Nogo66 inhibition, we investigated whether POSH mediates inhibition of axon outgrowth in response to Nogo66 in postnatal CGNs. Like cortical neurons, POSH RNAi CGNs were refractory to the inhibitory action of Nogo66 (supplemental Fig. S1, available at www.jneurosci.org as supplemental material), suggesting that a POSH-dependent mechanism operates in different neuronal cell types to inhibit axon outgrowth in response to Nogo66.

$\mathrm{POSH}$ regulates biological outcomes by assembling a protein interaction network, which includes the actin-myosin regulatory protein Shroom3 (Taylor et al., 2008) and the mixed lineage kinase LZK (Ikeda et al., 2001; Xu et al., 2003). To determine whether these known binding partners for $\mathrm{POSH}$ mediate myelin inhibition, primary cortical neurons were nucleofected with control, Shroom3, or LZK RNAi vectors and process outgrowth was analyzed in the presence or absence of myelin. Inhibition of Shroom 3 function by RNAi resulted in increased process length relative to control neurons when neurons were plated to PLL (Fig. $1 G$ ), consistent with our previous study demonstrating that the POSH-Shroom 3 complex inhibits process outgrowth (Taylor et al., 2008). Likewise, LZK RNAi cortical neurons exhibited increased process lengths when plated to PLL relative to control neurons (Fig. $1 \mathrm{H}$, supplemental Fig. S2 A, available at www. jneurosci.org as supplemental material), consistent with a role for LZK as a negative regulator of process outgrowth. Plating to myelin did not inhibit process length of Shroom3 or LZK RNAi neurons (Fig. 1G,H). In addition, process length was not decreased when Shroom3 or LZK RNAi neurons were plated to Nogo66 (Fig. 1G,H). Thus, reducing Shroom3 or LZK function with RNAi results in neurons that are refractory to myelin and Nogo66 inhibition. Together, the observations that POSH and its binding partners Shroom 3 and LZK modulate responsiveness to myelin and Nogo66 identify the POSH complex as an intracellular signal transducer for myelin-derived inhibitors.

Like NogoA (Huber et al., 2002; X. Wang et al., 2002; Mingorance-Le Meur et al., 2007), POSH is expressed in mouse embryonic cortical neurons in vitro (supplemental Fig. S3, available at www.jneurosci.org as supplemental material). Given this overlap in expression, RNAi-mediated reduction of NogoA function was used to investigate the potential for signaling from endogenous NogoA to POSH. Cortical primary neurons were nucleofected with control or Nogo-specific RNAi expression vectors to determine whether Nogo expression in cortical neurons limits axon outgrowth. The Nogo gene encodes for three variants, NogoA-C (Gonzenbach and Schwab, 2008), that share a C terminal neurite outgrowth inhibitory domain, Nogo66 (GrandPré et al., 2000; Oertle et al., 2003). In addition, NogoA has a unique domain, not present in NogoB or NogoC, that inhibits neurite outgrowth (Oertle et al., 2003). The Nogo-3 RNAi construct tar- 
gets the exon unique to NogoA and thus this construct specifically targets NogoA (supplemental Fig. S4, available at www. jneurosci.org as supplemental material). Process length in Nogo-3 RNAi-depleted neurons was increased relative to control (Fig. 2A), as expected if NogoA limits process outgrowth. Similar results were obtained with a second siRNA, Nogo-1, that targets the 3'UTR of the Nogo mRNA, reducing the expression of Nogo isoforms, NogoA/B (supplemental Fig. S4, available at www.jneurosci.org as supplemental material). The Nogo-1 RNAi construct is also expected to reduce the expression of NogoC because the $3^{\prime}$ UTR is conserved among the mRNAs for the three Nogo isoforms and RT-PCR analysis indicates that NogoC, along with $\operatorname{Nogo}_{0} / \mathrm{B}$, is expressed in cortical neurons (supplemental Fig. S3, available at www.jneurosci.org as supplemental material). Together, these results support a role for NogoA as an inhibitor of process outgrowth of cortical neurons grown in culture, and suggest that NogoB and NogoC do not function redundantly with NogoA to regulate process outgrowth. Since RNAi-mediated reduction of NogoA function in neurons enhances process length, this result suggests that NogoA acts in a cell autonomous, intrinsic fashion to inhibit process outgrowth. In addition, NogoA can act in a non-cell autonomous fashion, as process outgrowth of control cortical neurons is inhibited when plated directly onto purified Nogo66 protein (Fig. 1). Finally, purified Nogo66 protein, added externally, reverses the NogoA RNAi phenotype (Fig. 2C), unlike the addition of Nogo66 to PirB RNAi neurons (compare Figs. $2 \mathrm{C}, 1 \mathrm{H}$ ), indicating that cells in which NogoA is reduced can still respond to external Nogo66 as expected.

Myosin IIA RNAi reverses the increase in process length associated with RNAi depletion of POSH or the POSH-associated proteins Shroom3 (Taylor et al., 2008) and LZK (supplemental Fig. S2 B, available at www.jneurosci.org as supplemental material). However, myosin IIA RNAi does not reverse the increase in process length resulting from RNAi-mediated reduction of the neurite outgrowth inhibitors Robo1 or Ephrin B2 (Taylor et al., 2008). The molecular mechanism by which a reduction in myosin IIA function suppresses axon outgrowth in neurons deficient in POSH function, but not Ephrin B2 or Robo1, remains to be determined. Nonetheless, these observations suggest that myosin IIA reduction of function can distinguish among pathways that negatively regulate axon outgrowth, with some pathways sensitive to myosin IIA reduction of function (e.g., POSH) and others insensitive (Ephrin B2, Robo), and this provides a useful tool to assess whether specific components might be associated with a POSH signaling pathway for axon outgrowth inhibition. The ability of myosin IIA RNAi to suppress axon outgrowth was used to test whether NogoA signals to POSH to regulate process outgrowth. Primary cortical neurons were
B
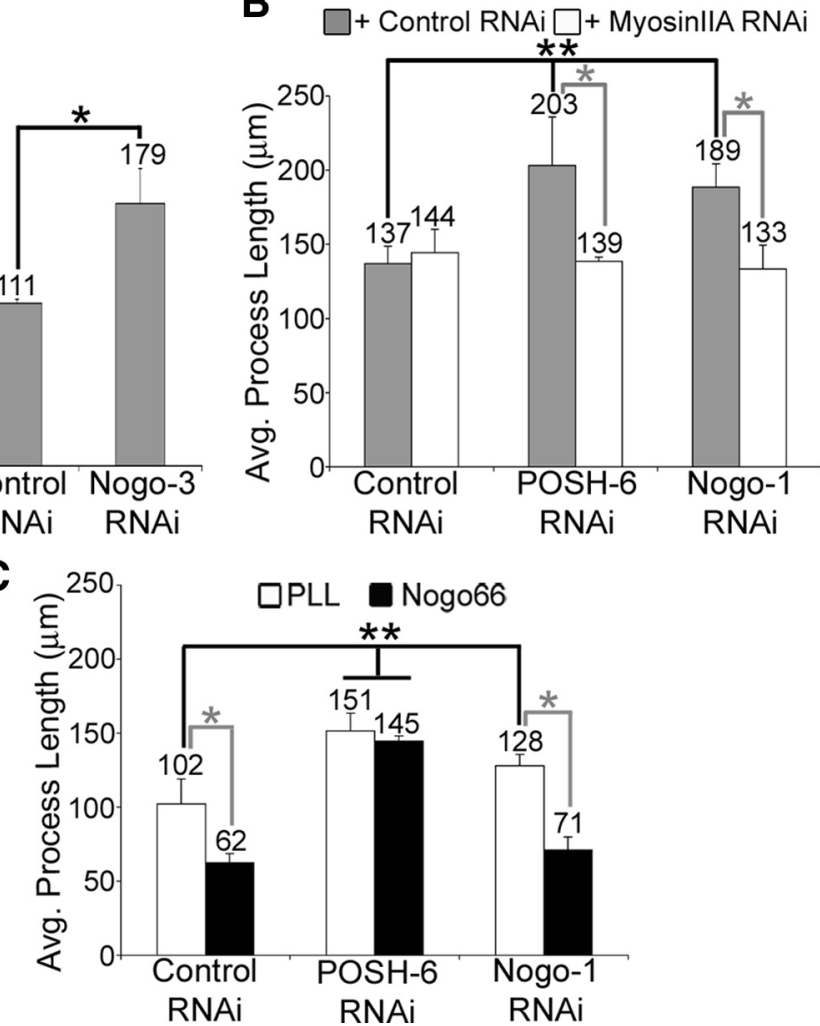

RNAi RNAi RNAi

Figure 2. Nogo inhibits axon outgrowth in cortical neurons in both a cell-autonomous and non-cell-autonomous fashion. $\boldsymbol{A}-\boldsymbol{C}$,

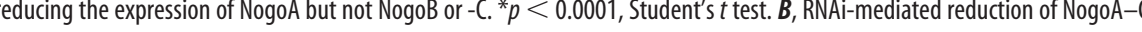
0.0001 , Student's $t$ test. $C$, External addition of purified Nogo66 reverses the Nogo RNAi phenotype. Process length was determined $3 \mathrm{~d}$ after nucleofection and plating to PLL or PLL plus Nog066. ${ }^{* * *} p<0.0001$, Student's $t$ test.

nucleofected with control, POSH, or Nogo RNAi expression vectors, together with a myosin IIA RNAi expression vector. As reported previously (Taylor et al., 2008), control neuron process length was unaffected by myosin IIA RNAi, and POSH RNAi neuron process length was reduced by myosin IIA RNAi (Fig. $2 B$ ). Process length of Nogo RNAi neurons was also reduced by myosin IIA RNAi (Fig. $2 B$ ), consistent with the hypothesis that NogoA signals through a POSH-dependent pathway to regulate process outgrowth.

Next, suppression analysis was used to test the hypothesis that LZK is a functional effector for Nogo signaling and to order the genes in the signaling pathway. If increased expression of LZK can compensate for loss of Nogo function, then this observation would support a role for LZK as a downstream effector of Nogo signaling. Cortical neurons were nucleofected with a Nogo RNAi expression vector and a control vector or a vector that expresses catalytically active or inactive forms of LZK. The efficiency of nucleofection of both plasmids was $94 \%$ (see Materials and Methods). Ectopic expression of wild-type, but not catalytically inactive, LZK suppressed the enhanced process length phenotype exhibited by Nogo RNAi nucleofected neurons (Fig. 3A-E). Suppression of the Nogo RNAi knockdown phenotype by increased expression of LZK is consistent with a model in which Nogo/ POSH signaling is mediated, at least in part, by LZK. It is also 
A
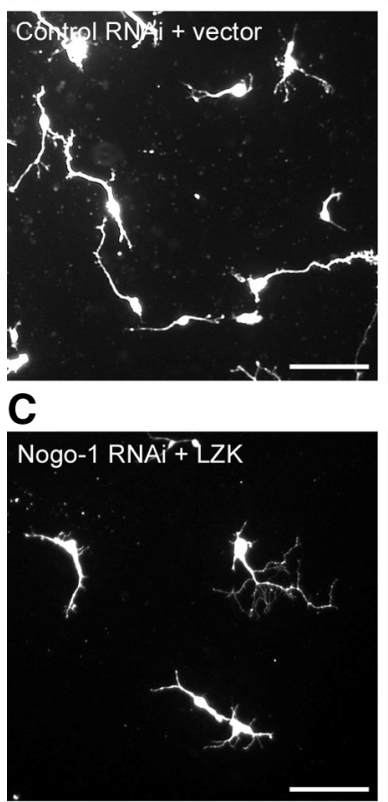

B
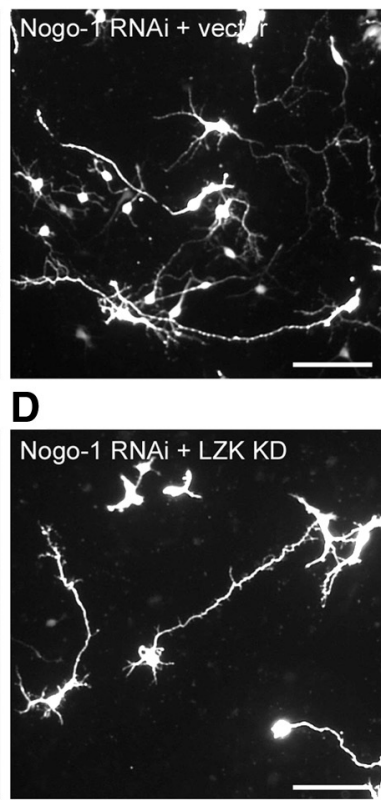

E
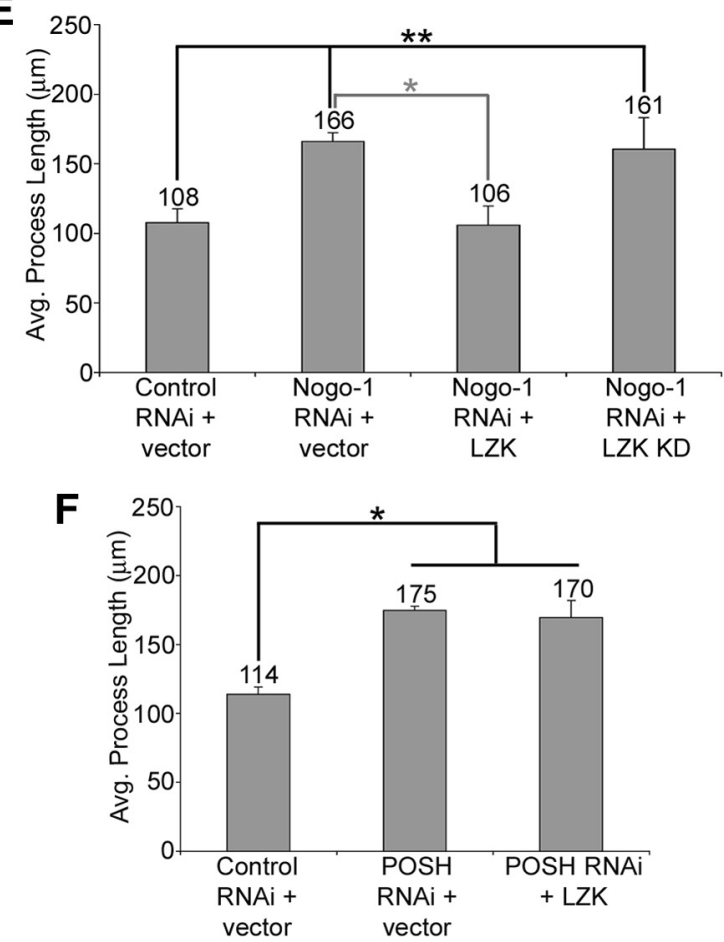

Figure 3. LZK is a downstream effector of a Nogo/POSH signaling pathway. A-E, LZK functions downstream of Nogo. Cortical neurons were nucleofected with the indicated RNAivectors and expression vectors for wild-type LZK, kinase dead (KD) LZK, or vector control. Nucleofected neurons were fixed and stained for GFP $72 \mathrm{~h}$ afternucleofection. Measurements were made on 237-312 neurons per condition from three independent experiments. The increase in process length from Nogo RNAi is reversed by ectopic expression of catalytically active, but not kinase dead, LZK. ${ }_{\text {, }}^{* *} p<0.0001$, Student's $t$ test. Scale bar, $100 \mu \mathrm{m}$. F, LZK suppression is POSH-dependent. Primary neurons were nucleofected with control or POSH RNAi vectors and an expression vector for wild-type LZK or vector control. In total, 325-414 neurons were measured per condition from three independent experiments. Ectopic expression of catalytically active LZK fails to reverse the POSH RNAi-mediated increase in process length. ${ }^{*} p<0.0001$, Student's $t$ test.

possible that increased LZK activity suppresses by activating a compensatory signaling pathway, not directly linked to NogoA function. However, this is unlikely because increased expression of LZK did not suppress process outgrowth of POSH RNAi neu- rons (Fig. 3F), indicating that LZK suppression of process outgrowth requires the POSH scaffold. Together, these observations indicate that LZK regulates process outgrowth inhibition in a POSH-dependent manner downstream of NogoA.

PirB is a functional receptor for Nogo and MAG in CGNs and dorsal root ganglion neurons (Atwal et al., 2008). If PirB transmits axon outgrowth inhibitory signals in cortical neurons, then reducing PirB function with RNAi should lead to increased process length. Cortical neurons with an RNAi-mediated reduction in PirB function exhibited an increase in process length (Fig. $4 \mathrm{~A}$ ) consistent with PirB functioning as a negative regulator of process outgrowth. A second RNAi construct, targeting a different PirB sequence, gave a similar result (data not shown and supplemental Fig. S4, available at www.jneurosci.org as supplemental material). Further, if PirB is acting as a receptor to transmit inhibitory signals from myelin and NogoA in cortical neurons, then PirB RNAi neurons should be refractory to myelin and Nogo66 inhibition. Process length of PirB RNAi neurons was unaffected by myelin and Nogo66 (Fig. $1 H$ ), supporting a role for PirB in myelin/Nogo-mediated inhibition in cortical neurons. Moreover, if PirB acts via POSH to inhibit process outgrowth, then myosin IIA RNAi is expected to reverse the PirB RNAi phenotype. Indeed, PirB RNAi neuronal process length was decreased by RNAi-mediated reduction in myosin IIA (Fig. 4B). Finally, if PirB signals to the POSH complex, then overexpression of LZK is expected to reverse the PirB RNAi phenotype. Overexpression of LZK in PirB RNAi-depleted cortical neurons reduced process length to control levels (Fig. $4 \mathrm{~A}$ ), supporting a role for the PirB receptor in transmitting inhibitory cues to the POSH/LZK complex to regulate process outgrowth.

\section{Discussion}

Together, these results define a central role for the POSH complex in relaying process outgrowth inhibition downstream of NogoA and PirB (Fig. 4C). Supporting a role for POSH as a downstream effector of myelin-based inhibitory signals, RNAimediated reduction in POSH function results in primary cortical neurons that robustly extend processes in the presence of CNS myelin, in contrast to control neurons, which are inhibited by myelin. As a scaffold protein, POSH operates through coassociating binding partners. POSH interacts with the actin-myosin regulatory protein Shroom 3 to regulate process outgrowth inhibition (Taylor et al., 2008) and we demonstrate here that neurons with RNAi-knockdown of Shroom3 function are refractory to the inhibitory action of CNS myelin or Nogo66, supporting a role for the POSH/Shroom3 complex in process outgrowth inhibition downstream of myelin and Nogo66. Neurons with an RNAimediated reduction in a second POSH interacting protein, the mixed lineage kinase LZK, are also refractory to the inhibitory action of myelin or Nogo66, providing further support for a key role for the POSH complex in transmitting axon outgrowth inhibitory cues from CNS myelin-derived inhibitors.

PirB is a receptor for Nogo66 (Atwal et al., 2008; Filbin, 2008) and our observations are consistent with PirB signaling to POSH to mediate process outgrowth inhibition in cortical neurons. First, RNAi-mediated reduction of PirB in primary cortical neurons results in increased neuronal process length and prevents inhibition by myelin or Nogo66, indicating that PirB, like POSH, negatively regulates process length in cortical neurons. Second, overexpression of the POSH-associated effector protein LZK suppresses the PirB RNAi-mediated increase in process length. Similarly, LZK suppresses increased process outgrowth from Nogo RNAi neurons. However, increased expression of LZK does 
not suppress the POSH RNAi enhanced process outgrowth phenotype, arguing that LZK does not suppress by simply activating a compensatory signaling pathway unlinked to PirB and POSH, but instead requires the POSH scaffold. Third, inhibition of myosin IIA function reverses the PirB RNAi phenotype, as well as the Nogo RNAi-mediated enhancement of process outgrowth. Similarly, myosin IIA RNAi reverses the increase in process length from LZK RNAi, as well as from POSH RNAi and Shroom3 RNAi (Taylor et al., 2008). The mechanism by which reduction of myosin IIA function is able to shorten process length under some conditions but not others (Taylor et al., 2008) remains to be determined. Nonetheless, that reduction of both ligand and receptor (Nogo/PirB) can be reversed by reduction of myosin IIA function, matching what is observed for POSH and $\mathrm{POSH}-$ associated proteins, further supports the proposed pathway of NogoA-PirB-POSH. Collectively, these observations implicate the PirB receptor as a critical component in signaling process outgrowth inhibition reliant on POSH function. In hematopoietic cells, PirB signals by recruitment of the tyrosine phosphatases SHP1/2 (Kubagawa et al., 1997; Ho et al., 1999). Likewise, in neurons responding to myelin or Nogo66 inhibition, PirB may recruit SHP2 to signal to POSH, but the identity of potential SHP2 substrate(s) in this pathway remains to be determined.

Further, these studies suggest that neuronally expressed NogoA limits axon outgrowth in a cell autonomous, intrinsic fashion, as RNAi-mediated reduction of

Nogo function in cortical neurons enhances axon outgrowth. In contrast to neurons in which PirB or POSH/Shroom3/LZK are inhibited by RNAi, external application of Nogo66 to Nogo RNAi knockdown neurons inhibits enhanced process outgrowth, consistent with the model that the NogoA RNAi phenotype represents loss of an autocrine ligand for PirB, whereas disruption of PirB or POSH complex function prevents inhibition by either extrinsic or autocrine Nogo. The existence of autocrine Nogo inhibition also suggests an explanation for increased process outgrowth from POSH RNAi neurons in the absence of external inhibitory molecules (Taylor et al., 2008). Intriguingly, multiple inhibitory domains reside within Nogo, yet Nogo66 alone is sufficient to suppress the Nogo RNAi phenotype, highlighting the important role of this domain in mediating process outgrowth inhibition. The ability of NogoA to limit process outgrowth in cis may function to limit axon outgrowth and plasticity during development (McGee et al., 2005; Syken et al., 2006; Mingorance-Le Meur et al., 2007; Atwal et al., 2008; Wang et al., 2008; Brosamle and Halpern, 2009; Datwani et al., 2009; Petrinovic et al., 2010). Collectively, these results indicate that extrinsic and intrinsic mechanisms for impeding axon outgrowth converge on the POSH scaffold complex. Whether POSH signaling is downstream of CNS axon outgrowth inhibitors, in addition to NogoA/
C

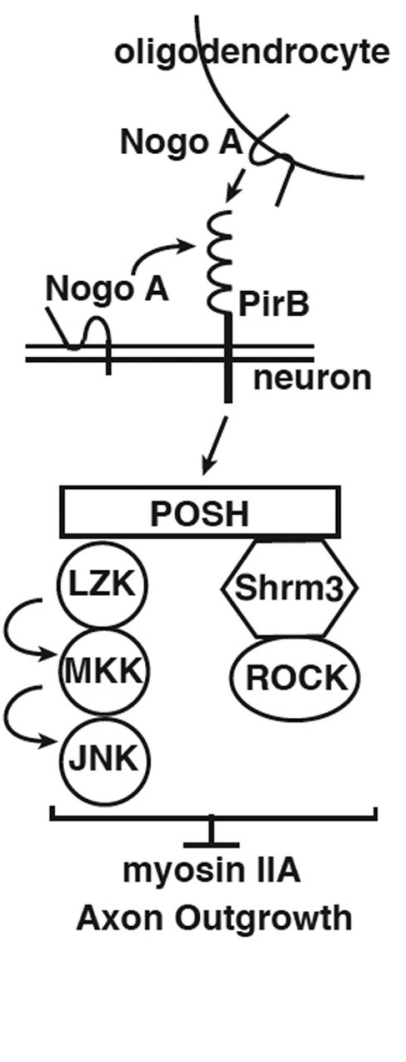

Figure 4. The PirB receptor transmits inhibitory signals from myelin and Nogo66 to the LZK-POSH scaffold complex. $\boldsymbol{A}, \boldsymbol{B}$, 作

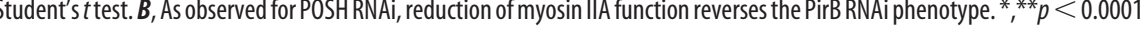

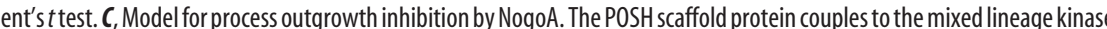
ectin-myosin regulatory protein Shroom 3 to relay process outgrowth inhibitory signals from NogoA. PirB, a receptor fo NogoA, relays signals to the POSH complex. External NogoA can inhibit axon outgrowth. In addition, NogoA on the neuron can self-limit axon outgrowth in a cell autonomous manner. MKK, mitogen-activated protein kinase kinase; JNK, c-Jun N-terminal kinase.

Nogo66, is an important question to be addressed in future studies.

Several Nogo isoforms are expressed in neurons, NogoA-C. All three isoforms contain the Nogo66 C-terminal inhibitory domain and are reported to localize to the plasma membrane (Dodd et al., 2005). Surprisingly, RNAi-mediated reduction of NogoA function alone is indistinguishable from RNAi-mediated reduction of all three Nogo isoforms simultaneously, indicating that NogoB and -C fail to compensate for NogoA deficiency. Perhaps unique sequences present in NogoA enable the protein to fold in a conformation that facilitates binding to a Nogo receptor residing in the same membrane (in cis).

Proteins present in CNS myelin, including NogoA, are a major impediment to the repair of the injured CNS. The results presented here demonstrate a novel function for the POSH scaffold in signaling process outgrowth inhibition in response to NogoA, and delineate a new signaling pathway for process outgrowth inhibition, comprised of NogoA, PirB, POSH, and LZK.

Identification of intracellular signaling components mediating myelin inhibition provides potential new targets for the development of therapeutics aimed at restoring function in the injured CNS. Blockade of POSH scaffold function, or the function of POSH comediators, has the potential to enhance axon 
outgrowth and plasticity and functional recovery in the injured CNS.

\section{References}

Atwal JK, Pinkston-Gosse J, Syken J, Stawicki S, Wu Y, Shatz C, TessierLavigne M (2008) PirB is a functional receptor for myelin inhibitors of axonal regeneration. Science 322:967-970.

Bilimoria PM, Bonni A (2008) Cultures of cerebellar granule neurons. Cold Spring Harb Protoc doi:10.1101.pdb.prot5107.

Brösamle C, Halpern ME (2009) Nogo-Nogo receptor signalling in PNS axon outgrowth and pathfinding. Mol Cell Neurosci 40:401-409.

Chung KH, Hart CC, Al-Bassam S, Avery A, Taylor J, Patel PD, Vojtek AB, Turner DL (2006) Polycistronic RNA polymerase II expression vectors for RNA interference based on BIC/miR-155. Nucleic Acids Res 34:e53.

Datwani A, McConnell MJ, Kanold PO, Micheva KD, Busse B, Shamloo M, Smith SJ, Shatz CJ (2009) Classical MHCI molecules regulate retinogeniculate refinement and limit ocular dominance plasticity. Neuron 64:463-470.

Dodd DA, Niederoest B, Bloechlinger S, Dupuis L, Loeffler JP, Schwab ME (2005) Nogo-A, -B, and -C are found on the cell surface and interact together in many different cell types. J Biol Chem 280:12494-12502.

Figueroa C, Tarras S, Taylor J, Vojtek AB (2003) Akt2 negatively regulates assembly of the POSH-MLK-JNK signaling complex. J Biol Chem 278:47922-47927.

Filbin MT (2008) PirB, a second receptor for the myelin inhibitors of axonal regeneration Nogo66, MAG, and OMgp: implications for regeneration in vivo. Neuron 60:740-742.

Fournier AE, GrandPre T, Strittmatter SM (2001) Identification of a receptor mediating Nogo-66 inhibition of axonal regeneration. Nature 409:341-346.

Giger RJ, Venkatesh K, Chivatakarn O, Raiker SJ, Robak L, Hofer T, Lee H, Rader C (2008) Mechanisms of CNS myelin inhibition: evidence for distinct and neuronal cell type specific receptor systems. Restor Neurol Neurosci 26:97-115.

Gonzenbach RR, Schwab ME (2008) Disinhibition of neurite growth to repair the injured adult CNS: focusing on Nogo. Cell Mol Life Sci 65:161-176.

GrandPré T, Nakamura F, Vartanian T, Strittmatter SM (2000) Identification of the Nogo inhibitor of axon regeneration as a Reticulon protein. Nature 403:439-444.

Ho LH, Uehara T, Chen CC, Kubagawa H, Cooper MD (1999) Constitutive tyrosine phosphorylation of the inhibitory paired Ig-like receptor PIR-B. Proc Natl Acad Sci U S A 96:15086-15090.

Huber AB, Weinmann O, Brösamle C, Oertle T, Schwab ME (2002) Patterns of Nogo mRNA and protein expression in the developing and adult rat and after CNS lesions. J Neurosci 22:3553-3567.

Ikeda A, Masaki M, Kozutsumi Y, Oka S, Kawasaki T (2001) Identification and characterization of functional domains in a mixed lineage kinase LZK. FEBS Lett 488:190-195.

Kubagawa H, Burrows PD, Cooper MD (1997) A novel pair of immunoglobulin-like receptors expressed by B cells and myeloid cells. Proc Natl Acad Sci U S A 94:5261-5266.

Kubo T, Hata K, Yamaguchi A, Yamashita T (2007) Rho-ROCK inhibitors as emerging strategies to promote nerve regeneration. Curr Pharm Des 13:2493-2499.

Larocca JN, Norton WT (2007) Isolation of myelin. Curr Protoc Cell Biol Chapter 3:Unit3.25.

Makeyev EV, Zhang J, Carrasco MA, Maniatis T (2007) The microRNA miR-124 promotes neuronal differentiation by triggering brain-specific alternative pre-mRNA splicing. Mol Cell 27:435-448.

McGee AW, Yang Y, Fischer QS, Daw NW, Strittmatter SM (2005) Experience-driven plasticity of visual cortex limited by myelin and Nogo receptor. Science 309:2222-2226.

Mingorance-Le Meur A, Zheng B, Soriano E, del Río JA (2007) Involvement of the myelin-associated inhibitor Nogo-A in early cortical development and neuronal maturation. Cereb Cortex 17:2375-2386.

Oertle T, van der Haar ME, Bandtlow CE, Robeva A, Burfeind P, Buss A, Huber AB, Simonen M, Schnell L, Brösamle C, Kaupmann K, Vallon R, Schwab ME (2003) Nogo-A inhibits neurite outgrowth and cell spreading with three discrete regions. J Neurosci 23:5393-5406.

Petrinovic MM, Duncan CS, Bourikas D, Weinman O, Montani L, Schroeter A, Maerki D, Sommer L, Stoeckli ET, Schwab ME (2010) Neuronal Nogo-A regulates neurite fasciculation, branching and extension in the developing nervous system. Development 137:2539-2550.

Robak LA, Venkatesh K, Lee H, Raiker SJ, Duan Y, Lee-Osbourne J, Hofer T, Mage RG, Rader C, Giger RJ (2009) Molecular basis of the interactions of the Nogo-66 receptor and its homolog NgR2 with myelin-associated glycoprotein: development of NgROMNI-Fc, a novel antagonist of CNS myelin inhibition. J Neurosci 29:5768-5783.

Schnorr JD, Holdcraft R, Chevalier B, Berg CA (2001) Ras1 interacts with multiple new signaling and cytoskeletal loci in Drosophila eggshell patterning and morphogenesis. Genetics 159:609-622.

Schwab ME, Caroni P (2008) Antibody against myelin-associated inhibitor of neurite growth neutralizes nonpermissive substrate properties of CNS white matter. Neuron 60:404-405.

Syken J, Grandpre T, Kanold PO, Shatz CJ (2006) PirB restricts oculardominance plasticity in visual cortex. Science 313:1795-1800.

Tapon N, Nagata K, Lamarche N, Hall A (1998) A new ractarget POSH is an SH3-containing scaffold protein involved in the JNK and NF-kappaB signalling pathways. EMBO J 17:1395-1404.

Taylor J, Chung KH, Figueroa C, Zurawski J, Dickson HM, Brace EJ, Avery AW, Turner DL, Vojtek AB (2008) The scaffold protein POSH regulates axon outgrowth. Mol Biol Cell 19:5181-5192.

Tuvia S, Taglicht D, Erez O, Alroy I, Alchanati I, Bicoviski V, Dori-Bachash M, Ben-Avraham D, Reiss Y (2007) The ubiquitin E3 ligase POSH regulates calcium homeostasis through spatial control of Herp. J Cell Biol 177:51-61.

Venkatesh K, Chivatakarn O, Lee H, Joshi PS, Kantor DB, Newman BA, Mage R, Rader C, Giger RJ (2005) The Nogo-66 receptor homolog NgR2 is a sialic acid-dependent receptor selective for myelin-associated glycoprotein. J Neurosci 25:808-822.

Vojtek AB, Taylor J, DeRuiter SL, Yu JY, Figueroa C, Kwok RP, Turner DL (2003) Akt regulates basic helix-loop-helix transcription factor-coactivator complex formation and activity during neuronal differentiation. Mol Cell Biol 23:4417-4427.

Walmsley AR, Mir AK (2007) Targeting the Nogo-A signalling pathway to promote recovery following acute CNS injury. Curr Pharm Des 13:2470-2484.

Wang J, Chan CK, Taylor JS, Chan SO (2008) The growth-inhibitory protein Nogo is involved in midline routing of axons in the mouse optic chiasm. J Neurosci Res 86:2581-2590.

Wang KC, Kim JA, Sivasankaran R, Segal R, He Z (2002) P75 interacts with the Nogo receptor as a co-receptor for Nogo, MAG and OMgp. Nature 420:74-78.

Wang X, Chun SJ, Treloar H, Vartanian T, Greer CA, Strittmatter SM (2002) Localization of Nogo-A and Nogo-66 receptor proteins at sites of axonmyelin and synaptic contact. J Neurosci 22:5505-5515.

Xu Z, Kukekov NV, Greene LA (2003) POSH acts as a scaffold for a multiprotein complex that mediates JNK activation in apoptosis. EMBO J 22:252-261.

Xu Z, Kukekov NV, Greene LA (2005) Regulation of apoptotic c-Jun $N$-terminal kinase signaling by a stabilization-based feed-forward loop. Mol Cell Biol 25:9949-9959.

Zhang QG, Wang R, Han D, Dong Y, Brann DW (2009) Role of Racl GTPase in JNK signaling and delayed neuronal cell death following global cerebral ischemia. Brain Res 1265:138-147. 